\title{
Pure gonadal dysgenesis misdiagnosed as Mayer Rokitansky Kuster Hauser Syndrome: a case report
}

\author{
Preeti B. Singh ${ }^{1}$, Roli Purwar ${ }^{1 *}$, Pawan Kumar ${ }^{2}$
}

\begin{abstract}
${ }^{1}$ Department of Obstetrics and Gynaecology, ${ }^{2}$ Department of Radiology, All India Institute of Medical Sciences, Gorakhpur, Uttar Pradesh, India
\end{abstract}

Received: 31 August 2020

Accepted: 06 October 2020

\author{
*Correspondence: \\ Dr. Roli Purwar, \\ E-mail: purwarroli@gmail.com
}

Copyright: (c) the author(s), publisher and licensee Medip Academy. This is an open-access article distributed under the terms of the Creative Commons Attribution Non-Commercial License, which permits unrestricted non-commercial use, distribution, and reproduction in any medium, provided the original work is properly cited.

\begin{abstract}
Gonadal dysgenesis is a group of heterogeneous disorders with very rare presentation. The spectrum of disease not only includes primary amenorrhoea but also secondary amenorrhoea. Herein, we are reporting a case of 16-year-old phenotypic female who presented with amenorrhoea with 46, XX karyotype with hypoplastic uterus with absent ovaries (on imaging), with high gonadotropins level and low estradiol. Suspecting Mayer-Rokitansky-Küster-Hauser syndrome (due to hypoplastic uterus) with gonadal dysgenesis she was started on cyclic hormones for development of secondary sexual characters and to prevent bone loss. But, during follow up, after giving estrogen for 8 months, her hypoplastic uterus again starts reappearing with attainment of cyclic menses on estrogen and progesterone withdrawal. We concluded that, in the presence of rudimentary or hypoplastic uterus, straightforward diagnosis of MRKH is to be avoided without seeing peripheral estrogenisation, hormone profile and karyotype analysis.
\end{abstract}

Keywords: 46-XX, Gonadal dysgenesis, Hypergonadotropic hypogonadism, Hypoplastic uterus, Mayer-Rokitansky-Kuster-Hauser syndrome, Amenorrhoea

\section{INTRODUCTION}

Gonadal dysgenesis is an infrequent cause for primary amenorrhea (less than 1:100 000). It constitutes group of anomalies in which gonads are completely absent or maldeveloped and there is progressive loss of germ cells in the developing gonads of an embryo. Around $75 \%$ cases are associated with whole (turner syndrome; 45, $\mathrm{XO})$ or partial deletion of the $\mathrm{X}$ chromosome [mosaics $45, X / 46, X X, 45, X / 46, X, \operatorname{dic}(X)]$ and $25 \%$ are due to pure gonadal dysgenesis i.e. $46 \mathrm{XX}, 46 \mathrm{XY}$.

46, XX pure gonadal dysgenesis is a rare form of gonadal dysgenesis with wide clinical presentation. It may present with Primary or secondary amenorrhoea with or without normal secondary sex characteristics. Mayer-Rokitansky-Küster-Hauser syndrome (MRKHS) is an important differential diagnosis in such cases affecting at least 1 out of 4500 women. It is characterised by congenital aplasia or hypoplasia of uterus, fallopian tubes, cervix and the upper part (2/3) of the vagina in women showing normal development of secondary sexual characteristics. There are few case reports in literature where the two disorders coexisted. Sometimes, it becomes difficult to differentiate whether the hypoplastic uterus is because of MRKHS or severe estrogen deficiency due to gonadal dysgenesis. ${ }^{1}$

Here, in we are describing an interesting case where initial diagnosis of gonadal dysgenesis coexisting with Mullerian agenesis was made, but later on it was found to be a case of pure gonadal dysgenesis only. 


\section{CASE REPORT}

A 16-year-old unmarried, sexually inactive girl presented to us with chief complaints of secondary amenorrhoea for 3 years and poor breast development. She attained spontaneous menarche at 13 years with single episode of bleeding lasting for 4 days following which she became amenorrhoic. She was born full term by normal vaginal delivery; with uneventful perinatal and neonatal period. Her medical and surgical history was insignificant. She was thin built, height $149 \mathrm{~cm}$ and weight $40.5 \mathrm{~kg}$, BMI being $18.24 \mathrm{~kg} / \mathrm{m}^{2}$, normal intelligence and school performance. There was no facial dysmorphism, neck swelling, or any skeletal abnormalities. Her axillary hairs were absent, breast was tanner stage 1 , and pubic hairs tanner stage 2 . Abdomen was soft with no organomegaly. On genital examination external genitalia was normal, with single finger examination, her vaginal length was normal around $8 \mathrm{~cm}$ and uterine nodule could not be felt by per rectal examination.

Her hormonal profile was suggestive of hypergonadotropic hypogonadism (luteinizing hormone: 32.79 IU/1; follicle stimulating hormone: 63 IU/1; Serum prolactin: 8.28 ; thryoid stimulating hormone: 4.2 , serum estradiol $<5 \mathrm{pg} / \mathrm{ml})$. Her ultrasound showed hypoplastic uterus $(3.2 \times 0.7 \mathrm{~cm})$, with endometrial thickness of $1-2$ $\mathrm{mm}$. Bilateral ovaries were absent with normal adnexa. Magnetic resonance imaging was also suggestive of hypoplastic uterus, with absent bilateral ovaries. Her karyotype was XX, 46, obtained from blood lymphocyte culture. Initial diagnosis of MRKHS with gonadal dysgenesis with no associated organ system abnormality was made. She was started on cyclic estrogen and progesterone to which she responded, but menses were scanty in nature lasting for short duration. Clinical reassessment was done after 8 months of cyclic hormone therapy, showing breast tanner stage 4 and pubic hairs tanner 3. Follow up ultrasound showed enlargement of uterus $(6.4 \times 1.8 \times 3.3 \mathrm{~cm})$ with endometrial thickness of $4.9 \mathrm{~mm}$ with uterine body-to-cervix ratio of $3: 1$. Seeing the response of uterus to estrogen, diagnosis was revised and final diagnosis of gonadal dysgenesis was made. Written informed consent was obtained from the patient for their anonymized information to be published in this article.

\section{DISCUSSION}

The genetic sex of an embryo is determined at fertilization. Bipotential gonads develop from the gonadal ridge, between 6-7 weeks of intrauterine life along with two sets of wolffian ducts (WD) and mullerian ducts (MD) developing from intermediate mesoderm. Y chromosome has testes determining effect (SRY gene). In the presence of SRY gene testes are formed and they cause regression of MD by secretion of Anti-mullerian hormone from sertoli cells. Androgens secreted from testes causes development of WD. Sex determination is irrespective of $\mathrm{X}$ chromosome, however two $\mathrm{X}$ chromosomes are necessary for ovary development. Absence of SRY gene causes indifferent gonads to develop into ovaries and MD persistence. MD causes development of uterus, fallopian tubes and upper $2 / 3 \mathrm{rd}$ of vagina. Androgen deficiency causes regression of WD. Thus, the development of female internal genitalia and development of ovaries are independent. ${ }^{2}$ Coexistence of MRKH with gonadal dysgenesis is very rare anomaly. Very few case reports exist in literature and also follow up of these patients was not reported after giving estrogen. Mostly the cases which have been reported, patients also had other systemic abnormality like spinal defect, cardiac defects, neurodevelopment defect and renal defect. ${ }^{1}$

Studies indicate that, there is potentially, functional defect in hormone receptors signalling in MRKHS. In a recent study by Brucker et al, they showed MRKH patients have an aberrant hormonal responsiveness resulting in impaired endometrial decidualization causing the reduced proliferative capacity of endometrium and the developmental defects associated with the syndrome. Reappearance of vanishing uterus after hormonal (estrogen) replacement possibly helps in ruling out diagnosis of MRKH. ${ }^{3}$ No comment on status of uterus can be said until adequate exposure to exogenous estrogen has been completed. Uterus could continue to grow to adult size with estrogen therapy. In terms of fertility, they may have conception and successful pregnancy with in vitro fertilization with donor oocytes. ${ }^{4}$

\section{CONCLUSION}

Patients with ovarian insufficiency, timely estrogen should be restarted, for secondary sexual characters development, to minimise bone loss and to achieve adequate uterine size for future fertility. In the presence of rudimentary or hypoplastic uterus, straightforward diagnosis of MRKH is to be avoided without seeing peripheral estrogenisation, hormone profile and karyotype analysis.

\section{ACKNOWLEDGMENTS}

We would like to thank the patient and her family for consenting to publish this case report.

\section{Funding: No funding sources \\ Conflict of interest: None declared \\ Ethical approval: Not required}

\section{REFERENCES}

1. Kisu I, Ono A, Iijma T, Katayama M, Iura A, Hirao N. Mayer-Rokitansky-Küster-Hauser syndrome with a uterine cervix and normal vagina associated with gonadal dysgenesis in a 46,XX female. J Obstet Gynaecol Res. 2019;45(7):1386-90.

2. Aatsha PA, Krishan K. Embryology, Sexual Development. In Stat Pearl. 2020 May 30. 
3. Brucker SY, Eisenbeis S, König J, Lamy M, Salker MS, Zeng N, et al. Decidualization is impaired in endometrial stromal cells from uterine rudiments in Mayer-Rokitansky-Küster-Hauser syndrome. Cellul Physiol Biochem. 2017;41(3):1083-97.

4. Thewjitcharoen Y, Veerasomboonsin V, Nakasatien S, Krittiyawong S, Himathongkam T. Misdiagnosis of Mullerian agenesis in a patient with 46, XX gonadal dysgenesis: a missed opportunity for prevention of osteoporosis. Endocrinol Diabet Metabol Cas Repo. 2019;2019(1).

Cite this article as: Singh PB, Purwar R, Kumar P. Pure gonadal dysgenesis misdiagnosed as Mayer Rokitansky Kuster Hauser Syndrome: a case report. Int J Reprod Contracept Obstet Gynecol 2020;9:4756-8. 